\title{
Suppression of nucleosome-binding protein 1 by miR-326 impedes cell proliferation and invasion in non-small cell lung cancer cells
}

\author{
DONGFAN LI ${ }^{1}$, XUSHENG DU ${ }^{1}, \mathrm{AN} \mathrm{LIU}^{1}$ and PENG LI ${ }^{2}$ \\ ${ }^{1}$ Department of Respiration Medicine, The Xi'an Central Hospital of Xi'an Jiaotong University; \\ ${ }^{2}$ Department of Pediatric Surgery, The Second Affiliated Hospital of Xi'an \\ Jiaotong University, Xi'an, Shaanxi 710004, P.R. China
}

Received September 6, 2015; Accepted October 14, 2015

DOI: 10.3892/or.2015.4403

\begin{abstract}
Emerging studies have proposed microRNAs (miRNAs) as novel therapeutic tools for cancer therapy. Nucleosome-binding protein 1 (NSBP1) has been suggested as an oncogene in various types of human cancers. The present study aimed to identify a novel miRNA that could directly target and negatively modulate NSBP1 expression. We found that NSBP1 was highly expressed in non-small cell lung cancer (NSCLC) cells, and knockdown of NSBP1 by NSBP1 small interfering RNA (siRNA) significantly suppressed NSCLC cell proliferation and invasion. Bioinformatics analysis revealed that miR-326 had a putative binding site within the 3'-untranslated region of NSBP1. Their substantial relationship was further verified by dual-luciferase reporter assay, real-time quantitative polymerase chain reaction and western blot analysis. Overexpression of miR-326 significantly inhibited NSCLC cell proliferation and invasion, which mimicked the effect of NSBP1 siRNA. Furthermore, suppression of NSBP1 by NSBP1 siRNA or miR-326 overexpression remarkably repressed the expression of cyclin $\mathrm{B} 1$ and matrix metalloproteinase 9 (MMP9), which are associated with cancer cell proliferation and invasion. Moreover, overexpression of NSBP1 obviously abolished the inhibitory effect of miR-326 on cyclin B1 and MMP9 expression. In addition, an inverse correlation between miR-326 and NSBP1 expression
\end{abstract}

Correspondence to: Dr Peng Li, Department of Pediatric Surgery, The Second Affiliated Hospital of Xi'an Jiaotong University, 157 West 5th Road, Xi'an, Shaanxi 710004, P.R. China

E-mail: lipeng_xajt@163.com

Abbreviations: miRs, microRNAs; UTR, untranslated region; NSBP1, nucleosome-binding protein; NSCLC, non-small cell lung cancer; MMP9, matrix metalloproteinase-9

Key words: NSBP1, miR-326, non-small cell lung cancer, cell proliferation, cell invasion levels was found in NSCLC clinical specimens. Our study demonstrated a direct target relationship between NSBP1 and miR-326 through which miR-326 inhibited cell proliferation and invasion of NSCLC cells. Thus, miR-326-NSBP1 is a promising candidate target for developing novel anticancer therapeutics for NSCLC.

\section{Introduction}

Lung cancer is the leading cause of cancer death among various types of malignant tumours, with the highest morbidity and mortality rates worldwide (1). According to tumour histologic features, lung cancer can be divided into several subtypes, of which non-small cell lung cancer (NSCLC) occupies $>80 \%$ of the total lung cancer cases (2). Although current therapies have achieved certain progress, the disease still shows poor outcomes and low five-year survival rates $(<15 \%)(3,4)$. Therefore, elucidating the pathological mechanism to provide a theoretical basis for developing potential and effective therapies for NSCLC is imperative.

In recent years, microRNAs (miRNAs), a class of 18-22 nucleotide-long small non-coding RNAs, have been recognised as novel regulators for gene expression; miRNAs post-transcriptionally modulate gene expression by targeting the 3'-untranslated region (UTR) of the targets $(5,6)$. Thus, miRNAs regulate various cellular processes and participate in the pathogenesis of many diseases $(7,8)$. Certain miRNAs are dysregulated in almost all cancer types; these miRNAs do not only contribute to the development and progression of cancers, but also represent novel targets for cancer therapy (9-11). Therefore, miRNAs can serve as novel diagnostic biomarkers and potential candidate therapeutic targets.

Nucleosome-binding protein 1 (NSBP1) (also named high-mobility group nucleosome-binding domain 5) has been widely studied because of its ability to modulate gene transcription by binding to chromatin (12). NSBP1 is broadly distributed in many tissues and is mainly located in the nucleus $(13,14)$. NSBP1 is currently recognised as a candidate oncogene in various cancers, including prostate cancer (15), gliomas (16), bladder cancer (17), breast cancer (18) and osteosarcoma (19). In addition, silencing of NSBP1 suppresses 
lung cancer cell proliferation (20). However, the molecular basis of NSBP1 in regulating lung cancer remains unclear. Nevertheless, NSBP1 may be a potential candidate target for developing cancer therapies, including lung cancer.

miRNAs are regulators for gene silencing, representing novel therapeutic targets. However, to date, the specific miRNAs that can target and regulate NSBP1 have not been recognised. Thus, the present study aimed to identify a novel miRNA that specifically targets and modulates NSBP1 expression in NSCLC. Bioinformatics analysis revealed that miR-326 had a putative binding site within the 3'-UTR of NSBP1. Their substantial relationship was further verified by dual-luciferase reporter assay, real-time quantitative polymerase chain reaction (RT-qPCR) and western blot analysis. As expected, suppression of NSBP1 by miR-326 overexpression mimicked the effect of NSBP1 silencing by small interfering RNA (siRNA) on NSCLC cell proliferation and invasion. Suppression of NSBP by miR-326 overexpression or NSBP1 siRNA both inhibited the expression levels of cyclin B1 and MMP9, which are associated with tumour cell proliferation and invasion. Further data indicated that miR-326 and NSBP1 expression levels were inversely correlated in NSCLC tissues. Taken together, our study suggested a novel and critical functional significance of miR-326 in NSCLC, and this miRNA was a promising therapeutic candidate for NSCLC by suppressing NSBP1.

\section{Materials and methods}

Cell cultures. Human NSCLC cell lines NCI-H157 and A549 were obtained from American Type Culture Collection (Manassas, VA, USA). Human keratinocyte cell line HaCaT and human embryonic kidney cell line HEK293T were purchased from Type Culture Collection of the Chinese Academy of Sciences (Shanghai, China). These cells were maintained in DMEM (Invitrogen, Carlsbad, CA, USA) plus $10 \%$ fetal calf serum (FCS; Gibco, Rockville, MD, USA) and $1 \%$ penicillin/streptomycin (Sigma, St. Louis, MO, USA) and maintained in a humidified incubator containing $95 \%$ air $/ 5 \% \mathrm{CO}_{2}$ at $37^{\circ} \mathrm{C}$.

$R T-q P C R$. Total RNA was extracted using miRNeasy mini kit (Qiagen, Dusseldorf, Germany) according to the manufacturer's instructions. For mRNA expression analysis, cDNA was reverse transcribed by M-MLV reverse transcriptase (Takara, Dalian, Shanghai). For miRNA expression analysis, cDNA was synthesised with miScript reverse transcription kit (Qiagen). Primer sequences were as follows: NSBP1, forward 5'-GCAGTCAGGCAGTGACTGCCTTCG-3' and reverse 5'-CCCTTTTCTGTGGCATCTTC-3'; GAPDH, forward 5'-CAGTCAGCCGCATCTTCTTTT-3' and reverse 5'-GTGACCAGGCGCCCAATAC-3'; miR-326, forward 5'-ACTGTCCTTCCCTCTGGGC-3' and reverse 5'-AATGGT TGTTCTCCACTCTCTCTC-3'; U6 small nuclear RNA, forward 5'-GCTTCGGCAGCACATATACTAAAAT-3' and reverse, 5'-CGCTTCACGAATTTGCGTGTCAT-3'. GAPDH was used as an internal control for mRNA quantification. U6 small nuclear RNA was used as an internal control for miRNA quantification. RT-qPCR analysis was performed using SYBR-Green Master Mix (Bio-Rad, Hercules, CA, USA). The relative gene expression was analysed using the $2^{-\Delta \Delta \mathrm{Ct}}$ method and normalised to the internal controls.

Western blot analysis. Cells were lysed in RIPA lysis buffer, and the protein concentration was measured using a BCA kit (Beyotime Biotechnology, Haimen, China). Equal amounts of proteins from different samples were separated on $12.5 \%$ sodium dodecyl sulphate (SDS) polyacrylamide gel electrophoresis. The separated protein bands on SDS polyacrylamide gel were electro-transferred onto a nitrocellulose membrane (Bio-Rad). After blocking with 5\% skim milk powder, the membrane was blotted with NSBP1, cyclin B1, MMP9 and GAPDH antibodies (Cruz Biotechnology, Inc., Santa Cruz, CA, USA) at $4^{\circ} \mathrm{C}$ overnight. Horseradish peroxidase-conjugated secondary antibodies (Santa Cruz Biotechnology, Inc.) diluted in 1:2,000 were added and incubated for $1 \mathrm{~h}$ at room temperature. Finally, the immunoreactive bands were developed with the enhanced chemiluminescence method. The grey value of protein bands was quantified with Image-Pro Plus 6.0 software (Media Cybernetics, Inc., Rockville, MD, USA), and relative protein expression data were normalised to GAPDH.

Cell transfection. NSBP1-specific siRNA and control non-specific siRNA (NC siRNA) were purchased from Cruz Biotechnology, Inc., and then transfected into cells according to the manufacturer's instructions. In brief, cells were plated into six-well plates at $2 \times 10^{5}$ cells/well overnight. About $1 \mu \mathrm{g}$ of siRNA was diluted into $100 \mu \mathrm{l}$ of transfection medium, and $6 \mu \mathrm{l}$ of transfection reagent was diluted into $100 \mu \mathrm{l}$ of transfection medium. Both solutions were then mixed together for $45 \mathrm{~min}$ at room temperature. The siRNA transfection reagent mixture was added to each well with $0.8 \mathrm{ml}$ of transfection medium and incubated for $7 \mathrm{~h}$. The medium was replaced with fresh normal growth medium and cultured for another $48 \mathrm{~h}$. The interfering efficiency was detected by western blot analysis. miR-326 mimics and non-specific controls (miR-NC) were obtained from Shanghai GenePharma Co., Ltd. (Shanghai, China). NSCLC cells were seeded in six-well plates overnight and then transfected with $50 \mathrm{nM}$ miR-326 mimics or miR-326 inhibitor using Lipofectamine 2000 (Invitrogen) according to the manufacturer's instruction. The transfection efficiency was subsequently detected by RT-qPCR analysis after transfection for $48 \mathrm{~h}$.

Cell proliferation assay. Cell proliferation of NSCLC cells was measured by the 3-(4,5-dimethyl-2-thiazolyl)-2,5-diphenyl-2-H-tetrazolium bromide (MTT) method. In brief, NSCLC cells were seeded into 96-well cell plates at a density of $1 \times 10^{5}$ cells/well and grown for $24 \mathrm{~h}$. The cells were then transfected with miR-NC or miR-326 mimics for $48 \mathrm{~h}$. The old medium was replaced by fresh medium containing $20 \mu \mathrm{l}$ of MTT ( $5 \mathrm{mg} / \mathrm{ml}$ in PBS; Sigma) and cultured for $4 \mathrm{~h}$. The medium was discarded, and $200 \mu \mathrm{l}$ of dimethyl sulphoxide was added to dissolve crystal formazan. The optical density (OD) of each reaction solution at $490 \mathrm{~nm}$ was measured with an enzyme immunoassay analyser (Bio-Tek Instruments, Winooski, VT, USA).

Cell invasion assay. Tumour cell invasion ability was detected by Transwell invasion assay. The Transwell filter was precoated 

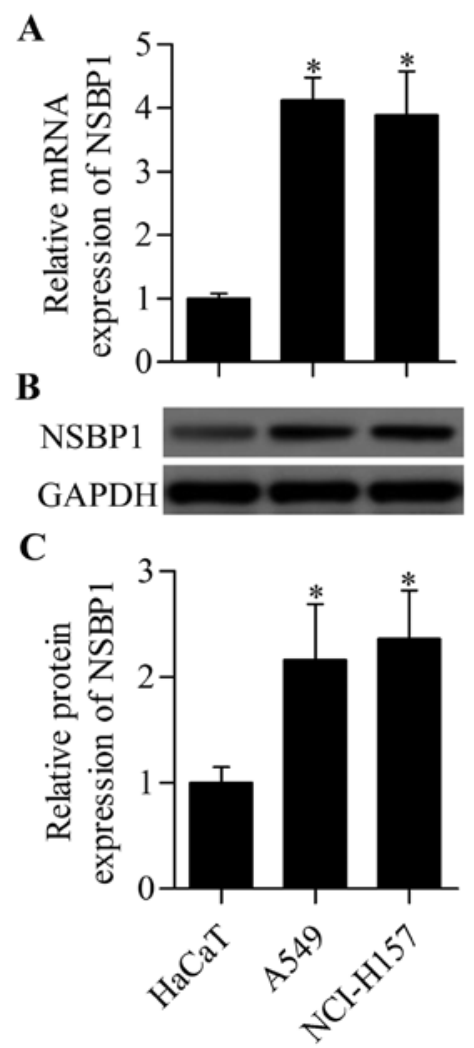

Figure 1. Biological effect of NSBP1 in human NSCLC cells. The mRNA (A) and protein (B) expression levels in NSCLC and A549 or NCI-H157 cells were detected by RT-qPCR and western blot analysis, respectively. (C) The relative expression of NSBP1 was quantified using Image-Pro Plus 6.0 software by normalisation with GAPDH. HaCaT cells served as the control. ${ }^{*} \mathrm{P}<0.05$ vs. HaCaT.

with Matrigel (BD Bioscience, San Jose, CA, USA) on the upper surface of a polycarbonic membrane. NSCLC cells were transfected with miR-326 mimics or miR-NC for $48 \mathrm{~h}$ and then starved overnight. A total of $1 \times 10^{5}$ cells were added in the top chamber with serum-free medium, and the medium containing $10 \%$ FCS was added to the lower chamber. After $48 \mathrm{~h}$, non-migrated cells in the upper chamber were removed by a cotton swab, and the migrated cells in the lower chamber were fixed with $95 \%$ ethanol and stained with $4 \mathrm{~g} / \mathrm{l}$ trypan blue solution. Cells were observed under a microscope (Olympus, Tokyo, Japan), and positive staining cells were counted in five random fields.

Cell cycle analysis. Cell cycle distribution was detected by flow cytometry. In brief, NSCLC cells were serum-starved for $24 \mathrm{~h}$ and transfected with NSBP1 siRNA or miR-326 mimics for $48 \mathrm{~h}$. Thereafter, cells were harvested, washed with ice-cold PBS and fixed with $70 \%$ ethanol. Propidium iodide containing RNase (Molecular Probes, Eugene, OR, USA) was added to the cells and incubated for $30 \mathrm{~min}$ in the dark. FACScan flow cytometry (Becton Dickinson, Franklin Lakes, NJ, USA) was used to detect the cell cycle distribution.

Dual-luciferase reporter assay. The cDNA fragments from NSBP1 containing an miR-326 binding site were inserted into pmirGLO vector (Promega, Madison, WI, USA). HEK293T cells were seeded into six-well plates at $2 \times 10^{5}$ cells/well overnight. About $10 \mathrm{ng}$ of pmirGLO-NSBP1 recombinant vectors and $50 \mathrm{nM}$ miR-326 mimics were introduced into HEK293T cells using Lipofectamine 2000 (Invitrogen). The transfected cells were lysed after $48 \mathrm{~h}$ of transfection. Firefly luciferase activity and Renilla luciferase activity were quantified by the dual-luciferase reporter method (Promega).

NSCLC specimen collection. Twenty pairs of NSCLC tissues and matched adjacent non-tumour tissues were provided by Xi'an Central Hospital of Xi'an Jiaotong University. The tissues were collected from NSCLC patients who had undergone surgical resections and stored at $-80^{\circ} \mathrm{C}$ for use. The use of clinical tissues was approved by the hospital's Institutional Human Experiment and Ethic Committee with informed consent from the patients.

Statistical analysis. Data are presented as the mean \pm standard deviation (SD). Statistical analyses were carried out using SPSS software (version 11.5; SPSS Inc., Chicago, IL, USA), and statistical differences were analysed using Student's t-test or one-way ANOVA. Correlation analysis was performed using Spearman's rank correlation coefficients. $\mathrm{P}<0.05$ was considered to indicate a statistically significant difference.

\section{Results}

NSBP1 is highly expressed in human NSCLC cells. To explore the potential role of NSBP1 in human NSCLC, the expression patterns of NSBP1 in the NSCLC cell lines were examined by RT-qPCR and western blot analysis. The results showed that the mRNA expression of NSBP1 was significantly higher in A549 and NCI-H157 cells than that in control HaCaT cells (Fig. 1A). Moreover, the NSBP1 protein expression was also highly upregulated in A549 and NCI-H157 cells (Fig. 1B and C). These results suggested a critical role of NSBP1 in NSCLC cells.

Knockdown of NSBP1 inhibits NSCLC cell proliferation and invasion. To understand the functional significance of NSBP1 in regulating the biological processes of NSCLC cells, we performed loss-of-function experiments of NSBP1 by transfection with specific siRNA targeting NSBP1. The transfection efficiency was detected by western blot analysis, which showed that NSBP siRNA effectively downregulated the expression of NSBP1 in A549 (Fig. 2A) and NCI-H157 (Fig. 2B) cells. Subsequently, we measured the effect of NSBP1 suppression on NSCLC cell proliferation. MTT assay showed that knockdown of NSBP1 significantly inhibited A549 (Fig. 2C) and NCI-H157 (Fig. 2D) cell proliferation. Furthermore, cell cycle distribution showed that knockdown of NSBP1 apparently induced G0/G1 cell cycle arrest of A549 (Fig. 3A) and NCI-H157 (Fig. 3B) cells. To further investigate the biological effect of NSBP1 in NSCLC cells, we examined the effect of NSBP1 knockdown on NSCLC cell invasion. We found that suppression of NSBP1 significantly repressed the invasive ability of A549 (Fig. 3C) and NCI-H157 (Fig. 3D) cells. In summary, these results indicated that NSBP1 participated in the regulation of NSCLC cell proliferation and invasion. 
A
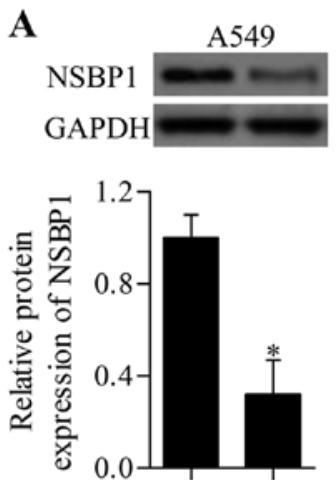

C

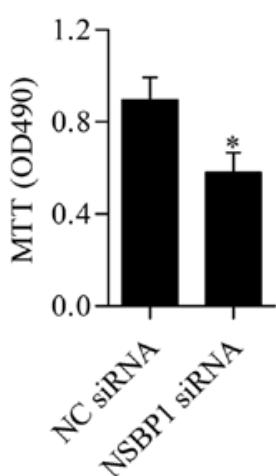

B

NSBP1

GAPDH

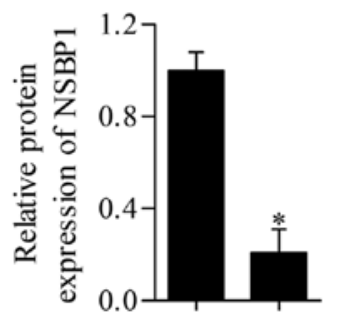

D

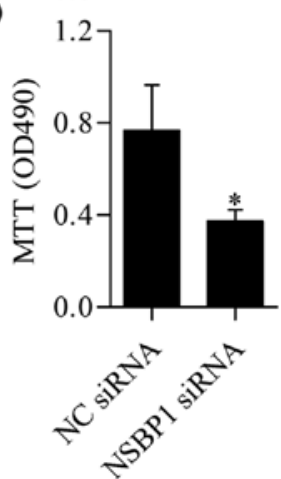

Figure 2. Knockdown of NSBP1 impairs NSCLC cell proliferation. The transfection efficiency of NSBP1 siRNA was detected by western blot analysis in A549 (A) and NCI-H157 (B) cells. NSBP1 siRNA was transfected into cell siRNA for $48 \mathrm{~h}$. NC siRNA was used as a control. The relative expression of NSBP1 was quantified using Image-Pro Plus 6.0. ${ }^{*} \mathrm{P}<0.05$ vs. NC siRNA. The effect of NSBP1 siRNA on cell proliferation of A549 (C) and NCI-H157 (D) cells detected by MTT assay. ${ }^{*} \mathrm{P}<0.05$ vs. NC siRNA.

miR-326 targets the 3'-UTR of NSBPI and regulates NSBPI expression. An increasing number of studies have suggested that miRNAs have the potential to treat lung cancer via targeting specific genes (21). To date, the miRNAs specifically targeting NSBP1 have not been well characterised. In the present study, we searched for specific miRNAs that could target and regulate NSBP, and investigated whether NSBP1 expression can be regulated by miRNAs. Bioinformatics analysis demonstrated that miR-326 had a predicted binding site in the 3'-UTR of NSBP1 (Fig. 4A). We then performed a dual-luciferase reporter experiment to verify their direct relationship. The results showed that overexpression of miR-326 markedly repressed the relative luciferase activity of pmirGLO-NSBP1, but it did not affect the relative luciferase activity of pmirGLO-NSBP1 (mut)-transfected cells (Fig. 4B).

To investigate whether miR-326 can regulate NSBP1 expression in NSCLC cells, we transfected miR-326 mimics into NSCLC cells and detected NSBP1 expression using RT-qPCR and western blot analysis. RT-qPCR analysis showed that miR-326 overexpression significantly suppressed the mRNA expression of NSBP1 in A549 (Fig. 4C) and NCI-H157 (Fig. 4D) cells. Western blot analysis further displayed that the protein expression of NSBP1 was also markedly repressed by miR-326 mimics in A549 (Fig. 4E) and NCI-H157 (Fig. 4F) cells. These results indicated that miR-326 could target the 3'-UTR of NSBP1 and repress its expression in NSCLC cells.

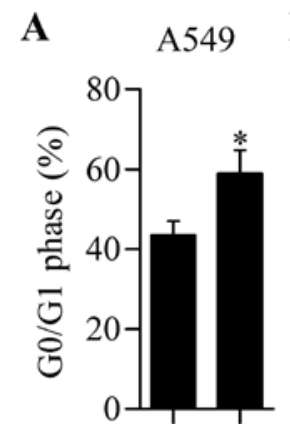

B $\mathrm{NCI}-\mathrm{H} 157$

C
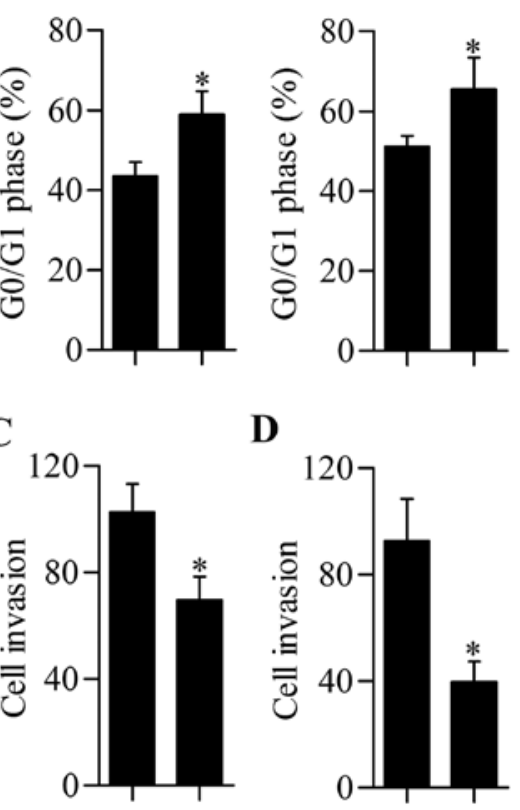

D
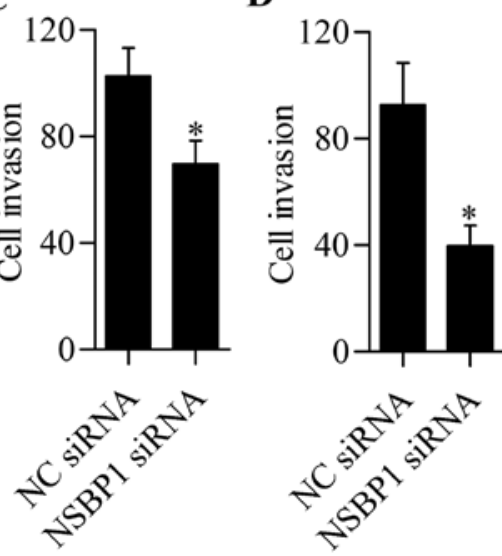

Figure 3. Knockdown of NSBP1 impairs NSCLC cell cycle progression and invasion ability. The effect of NSBP1 siRNA on cell cycle population in G0/G1 phase of A549 (A) and NCI-H157 (B) cells was measured by flow cytometry. ${ }^{*} \mathrm{P}<0.05$ vs. NC siRNA. The effect of NSBP1 siRNA on cell invasive ability of A549 (C) and NCI-H157 (D) was analysed by Transwell invasion assay. ${ }^{*} \mathrm{P}<0.05$ vs. $\mathrm{NC}$ siRNA.

Overexpression of miR-326 suppresses NSCLC cell proliferation and invasion. Given the inhibitory effect of miR-326 on NSBP1 expression, miR-326 might have a biological effect on NSCLC cells. To test this hypothesis, we examined the biological effect of miR-326 on cell proliferation and invasion in NSCLC cells. Interestingly, MTT assay demonstrated that transfection of miR-326 significantly inhibited cell proliferation of A549 (Fig. 5A) and NCI-H157 (Fig. 5B) cells. Moreover, miR-326 overexpression induced G0/G1 cell cycle arrest in A549 (Fig. 5C) and NCI-H157 (Fig. 5D) cells. The results of Transwell assay suggested that miR-326 mimic-transfected cells showed significantly decreased cell invasive capacity (Fig. 5E and F).

NSBP1 gene silencing induced by siRNA or miR-326 overexpression represses cyclin B1 and MMP9 in NSCLC cells. To further understand the molecular basis of NSBP1 gene silencing induced by NSBP1 siRNA or miR-326 in regulating NSCLC cell proliferation and invasion, we detected the regulatory effect of NSBP1 gene silencing on cyclin B1 and MMP9, which were involved in regulating cancer cell proliferation and invasion. We found that suppression of NSBP1 by siRNA or miR-326 inhibited the protein expression levels of cyclin B1 and MMP9 in A549 (Fig. 6A and B).

miR-326 regulates cyclin $B 1$ and MMP9 expression through NSBP1. To investigate whether miR-326 overexpression 


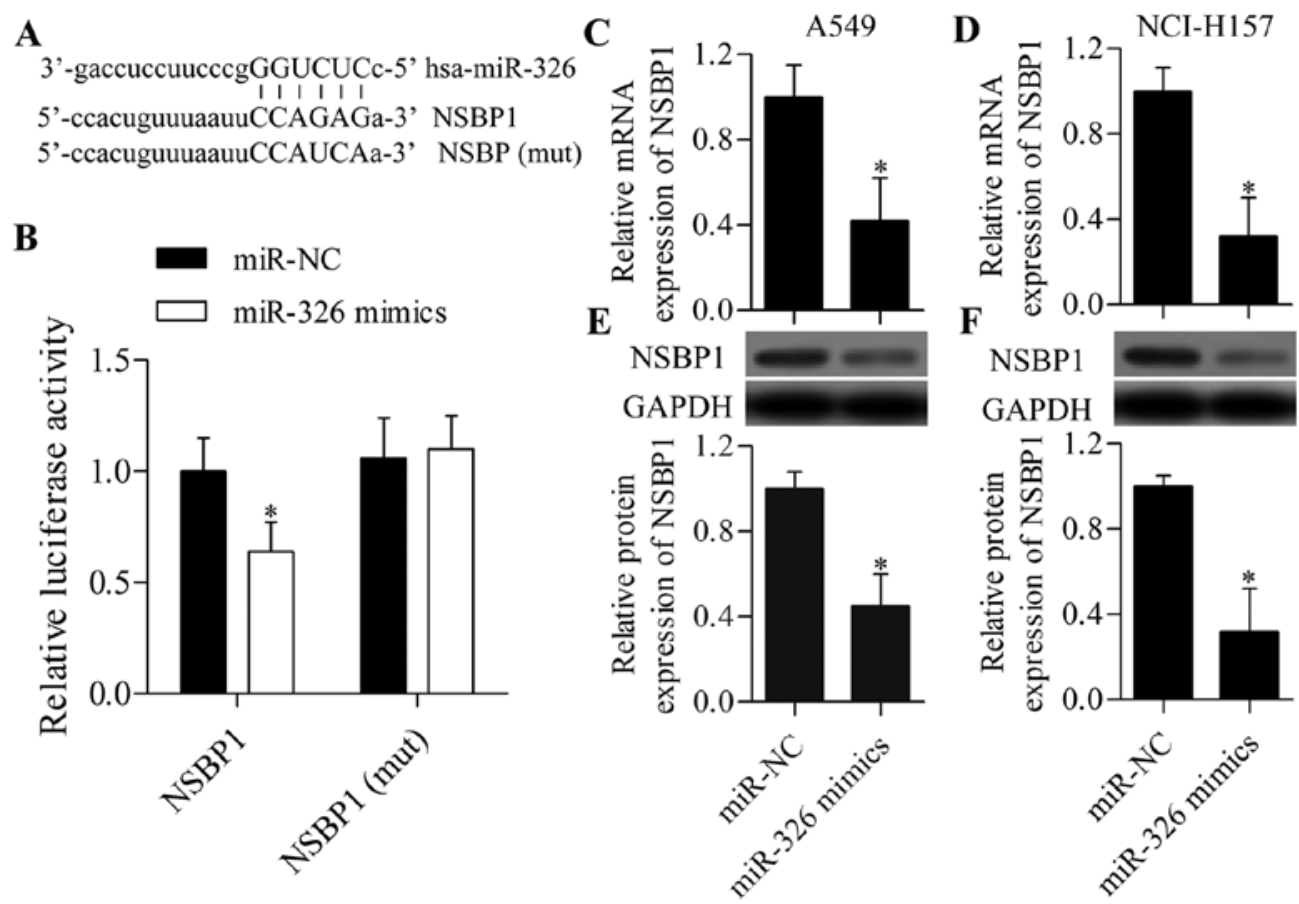

Figure 4. miR-326 targets and inhibits NSBP expression. (A) Putative miR-326 binding site in the 3'-UTR of NSBP1. (B) Luciferase reporter assay of miR-326 mimics and mirGLO vectors in HEK293T cells. "P<0.05 vs. miR-NC. RT-qPCR analysis of NSBP1 mRNA expression in A549 (C) and NCI-H157 (D) cells transfected with miR-326 mimics. "P<0.05 vs. miR-NC. Western blot analysis of NSBP1 protein expression in A549 (E) and NCI-H157 (F) cells transfected with miR-326 mimics. The relative protein expression was quantified by Image-Pro Plus 6.0. ${ }^{*} \mathrm{P}<0.05$ vs. miR-NC.
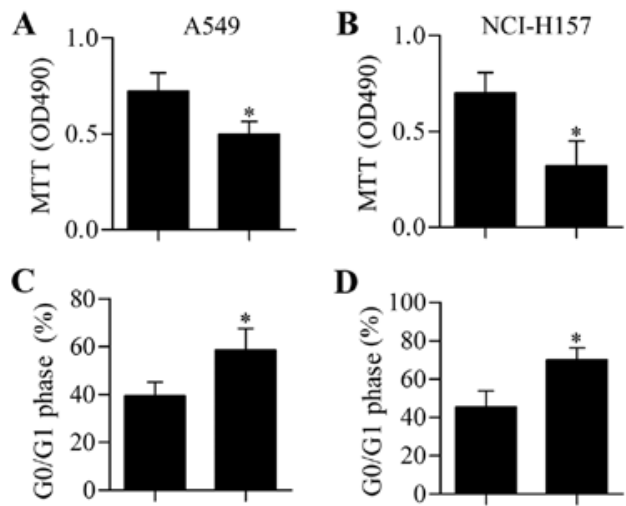

D
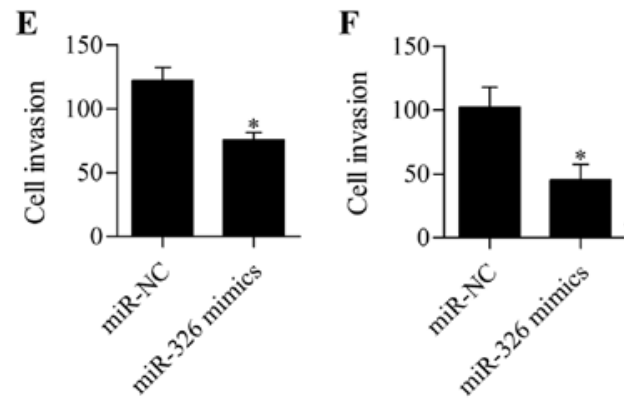

Figure 5. miR-326 inhibits NSCLC cell proliferation and invasion. MTT assay of cell proliferation in A549 (A) and NCI-H157 (B) cells transfected with miR-326 mimics. The effect of miR-326 mimics on cell cycle in A549 (C) and NCI-H157 (D) cells was detected by flow cytometry. The effect of miR-326 mimics on A549 (E) and NCI-H157 (F) cell invasion. " $\mathrm{P}<0.05$ vs. miRNA-NC.

suppressed cyclin B1 and MMP9 directly through NSBP1, we performed a rescue experiment by overexpression of NSBP1. We

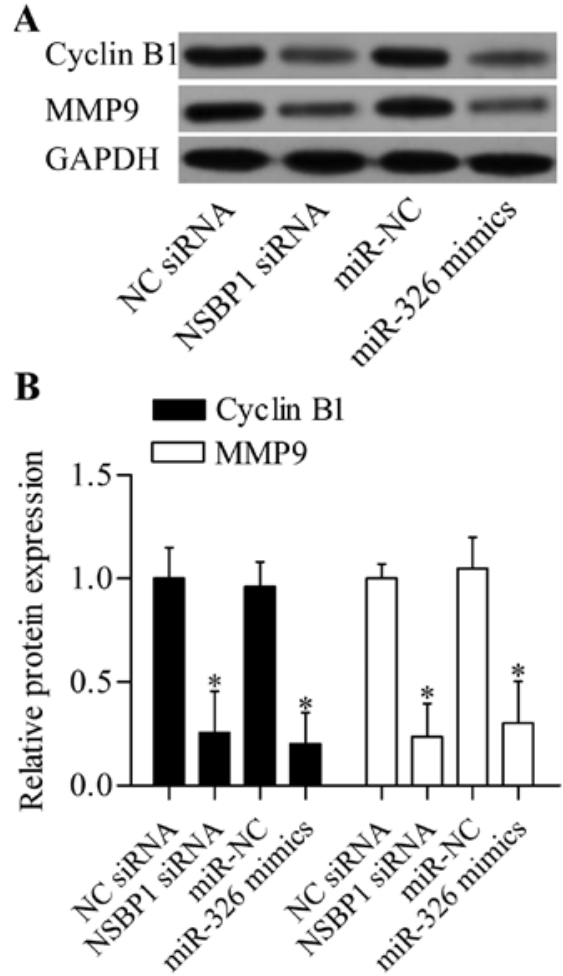

Figure 6. Suppression of NSBP1 represses cyclin B1 and MMP9. (A) Western blot analysis of cyclin B1 and MMP9 protein expression in A549 cells transfected with NSBP1 siRNA or miR-326 mimics. (B) Relative protein expression levels of cyclin B1 and MMP9 were quantitatively assessed by Image-Pro Plus 6.0. ${ }^{*} \mathrm{P}<0.05$ vs. NC siRNA or miR-NC.

found that transfection of NSBP expression vectors harbouring no 3'-UTR of NSBP1 significantly restored NSBP1 expression, 
A
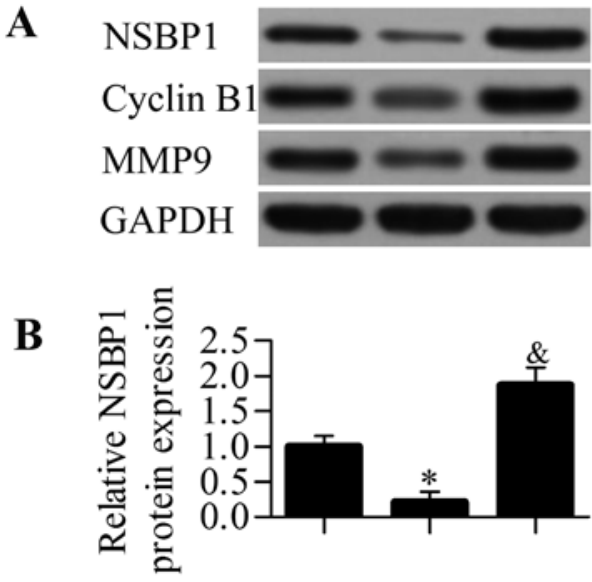

C

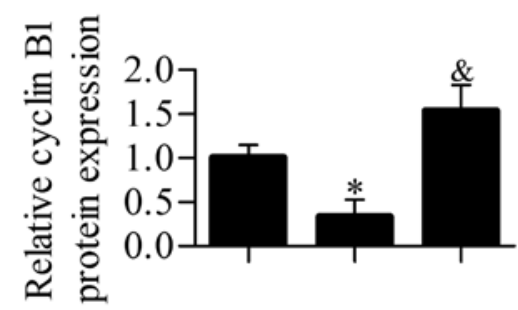

D

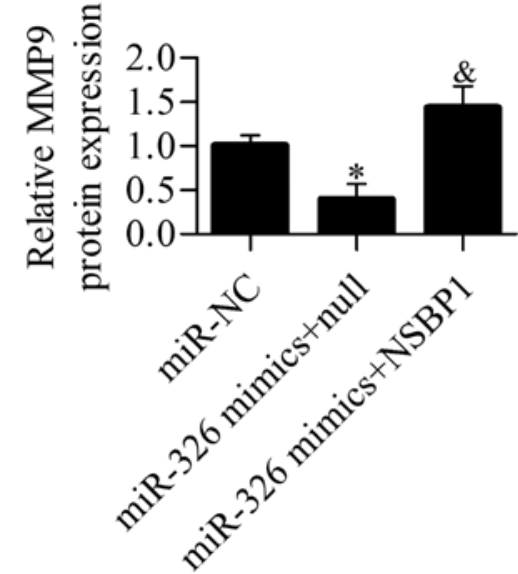

$\mathbf{E}$
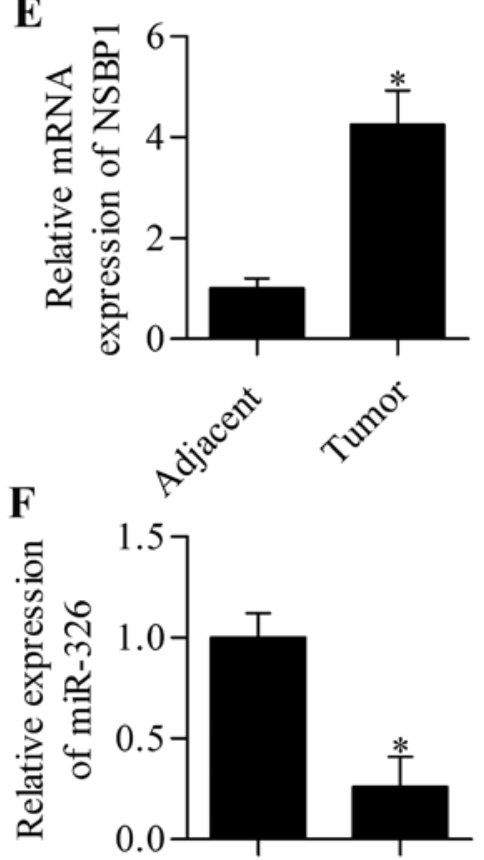

G

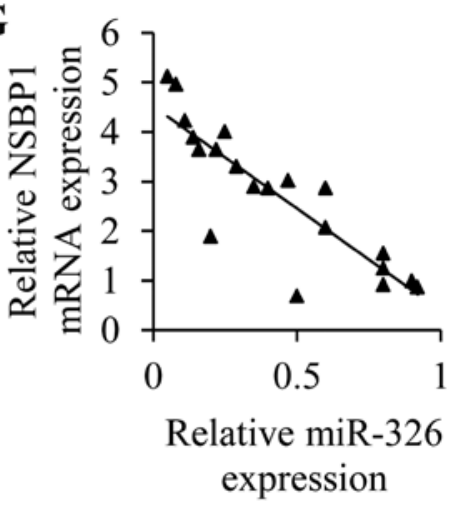

Figure 7. miR-326 regulates cyclin B1 and MMP9 through NSBP1 and the expression analysis of miR-326 and NSBP1 in clinical cancer tissues. (A) Western blot analysis of cyclin B1 and MMP9 protein expression in A549 cells co-transfected with miR-326 mimics and NSBP1-expressing vectors. The relative protein expression of NSBP1 (B), cyclin B1 (C) and MMP9 (D) was quantitated using Image-Pro Plus 6.0. Null, cells transfected with empty vectors; NSBP1, cells transfected with NSBP1-expressing vectors. ${ }^{\mathrm{P}}<0.05$ vs. miR-NC. ${ }^{\&} \mathrm{P}<0.05$ vs. miR-326 mimics + null. qPCR analysis of NSBP1 mRNA (E) and miR-326 (F) expression levels in 20 paired NSCLC tissues and adjacent non-tumour tissues. ${ }^{*} \mathrm{P}<0.05$ vs. adjacent. (G) Correlation of miR-326 expression with NSBP1 expression in NSCLC specimens using Spearman's correlation analysis. $r=-0.8756, P<0.001$.

which was decreased by the transfection of miR-326 mimics (Fig. 7A and B). NSBP1 overexpression restored the decreased protein expression levels of cyclin B1 and MMP9 induced by miR-326 overexpression (Fig. 7A, C and D), indicating that miR-326 inhibited cyclin B1 and MMP9 expression through NSBP1.

miR-326 expression is inversely correlated with NSBPI expression in NSCLC specimens. To gain insight into the functional significance of miR-326 and NSBP1 expression in NSCLC, we uncovered their expression levels in clinical NSCLC tissues and elucidated their potential relationship. We found that NSBP1 mRNA expression was significantly increased in NSCCL specimens (Fig. 7E), whereas miR-326 expression was markedly decreased (Fig. 7F) compared with that in adjacent non-tumour tissues. We correlated NSBP1 mRNA expression and miR-326 expression using correlation analysis, which demonstrated that miR-326 expression was remarkably inversely correlated with NSBP1 expression in NSCLC tissues (Fig. 7G) $(\mathrm{r}=-0.8756, \mathrm{P}<0.001)$, implying that decreased miR-326 might contribute to the increased NSBP1 expression during NSCCL development and progression.

\section{Discussion}

The present study showed that suppression of NSBP1 by miR-326 could inhibit NSCLC cell proliferation and invasion. We demonstrated that NSBP1, which functioned as an oncogene 
in NSCLC, could be targeted by miR-326. Overexpression of miR-326 significantly inhibited NSBP1 expression, which was analogous to NSBP1 siRNA. Our study suggested that miR-326 was a novel therapeutic target for treating NSCCL by suppressing NSBP1.

NSBP1 plays a critical role in modulating gene transcription (12). Thus, dysregulation of NSBP1 may be associated with disease generation and development. Increasing evidence revealed that NSBP1 is extensively involved in tumourigenesis. Jiang et al reported that knockdown of NSBP1 by RNA interference inhibits prostate cancer cell proliferation in vitro and in vivo (15). Knockdown of NSBP1 causes cell cycle arrest and promotes cell apoptosis of prostate cancer cells possibly by inhibiting cyclin $\mathrm{B} 1$ and $\mathrm{Bcl}-2$ expression levels (15). Similarly, Zhang et al showed that NSBP1 gene silencing results in increased mitochondria-mediated cell apoptosis in prostate cancer cells (22). Furthermore, NSBP1 is overexpressed in glioma clinical tissues, and knockdown of NSBP1 induces cell cycle arrest in the G1 phase, inhibits cell proliferation and promotes cell apoptosis in glioma cells in vitro (16). Moreover, NSBP1 was demonstrated to be overexpressed in bladder cancer (17), clear cell renal cell carcinoma (23), osteosarcoma (19) and breast cancer (18). These studies also reported that NSBP1 regulated cancer cell proliferation, apoptosis and invasion. In the present study, we found that NSBP1 was highly expressed in NSCLC cells, and knockdown of NSBP1 by NSBP1 siRNA significantly inhibited cell proliferation and invasion of NSCLC cells. Furthermore, knockdown of NSBP1 suppressed cyclin B1 and MMP9 expression levels, which might be the molecular basis for NSBP1 knockdown-induced decreased cell proliferation and invasion of NSCLC cells. Our results were consistent with the findings of Chen et al, who reported that knockdown of NSBP1 by siRNA inhibits cell proliferation and induces cell cycle arrest in the G1 phase in lung cancer cells (20). Notably, high expression of NSBP1 was also found to be associated with drug resistance and ionising radiation in cancer cells $(24,25)$. Collectively, our data, as well as the aforementioned findings, support the notion that NSBP1 could be a candidate target for developing cancer therapies.

Recent emerging studies have proposed miRNAs as novel therapeutic tools for cancer treatment by negatively modulating target gene expression $(5,6,9-11)$. These reports prompted us to identify specific miRNAs that could target and regulate NSBP1 expression. Intriguingly, we found that miR-326 possessed a predicted binding site in the 3 '-UTR of NSBP1, implying that miR-326 may directly modulate NSBP1 expression. Dual-luciferase activity validated the interaction between miR-326 and NSBP1 3'-UTR. Further experiments using RT-qPCR and western blot analysis confirmed that miR-326 inhibited NSBP1 expression in NSCLC cells. Strikingly, overexpression of miR-326 markedly inhibited cell proliferation and invasion of NSCLC cells, which mimicked the effect of NSBP1 siRNA, suggesting that miR-326 exerted a tumour suppressive function by inhibiting NSBP1 expression. Several studies have reported the potential therapeutic function of miR-326 against tumours. Kefas et al revealed that transfection of miR-326 significantly decreases cell growth and cell tumourigenicity of glioma cells $(26,27)$. Similarly, miR-326 expression is low in glioma tissues, and low miR-326 expression is associated with advanced pathological grade of gliomas (28). In pancreatic ductal adenocarcinoma, miR-326 significantly decreased in patients, and patients with relatively high miR-326 expression displayed long-term survival or without venous invasion, implying that miR-326 is a tumour suppressor in pancreatic ductal adenocarcinoma (29). Moreover, miR-326 was shown to exert a tumour suppressive role in glioma (30) or colorectal cancer (31) by targeting the Nin one binding protein that can inhibit cancer cell proliferation, migration and invasion, and promote cell apoptosis. Interestingly, miR-326 was found to suppress cancer cell invasion by targeting the disintegrin and metalloprotease 17 in lung adenocarcinoma (32). In line with these findings, we also found that miR-326 was frequently downregulated in NSCLC tissues, and overexpression of miR-326 inhibited cancer cell proliferation and invasion. These findings suggested that miR-326 functioned as a tumour suppressor.

Taken together, our study demonstrated a direct target relationship between NSBP1 and miR-326 through which miR-326 inhibited cell proliferation and invasion of NSCLC cells. Loss of NSBP1 induced by NSBP1 siRNA or miR-326 overexpression repressed the expression levels of cyclin B1 and MMP9, which contribute to cancer cell proliferation and invasion. Conversely, overexpression of NSBP1 apparently rescued the decreased cyclin B1 and MMP9 expression levels induced by miR-326 overexpression, further confirming that miR-326 exerted its tumour suppressive function by targeting NSBP1. Most importantly, we observed that miR-326 expression was inversely correlated with NSBP1 expression in NSCLC clinical specimens, further confirming its functional significance in NSCLC. Therefore, our study suggested that miR-326-NSBP1 could be employed as a promising candidate target for developing novel anticancer therapeutics for NSCLC.

\section{References}

1. Siegel R, Ma J, Zou Z and Jemal A: Cancer statistics, 2014. CA Cancer J Clin 64: 9-29, 2014.

2. Travis WD, Brambilla E, Noguchi M, Nicholson AG, Geisinger R Yatabe Y, Beer DG, Powell CA, Riely GJ, Van Schil PE, et al: International Association for the study of lung cancer/American Thoracic Society/European Respiratory Society International multidisciplinary classification of lung adenocarcinoma. J Thorac Oncol 6: 244-285, 2011.

3. Claassens L, van Meerbeeck J, Coens C, Quinten C, Ghislain I, Sloan EK, Wang XS, Velikova G and Bottomley A: Health-related quality of life in non-small cell lung cancer: An update of a systematic review on methodologic issues in randomized controlled trials. J Clin Oncol 29: 2104-2120, 2011.

4. Hu Z, Chen X, Zhao Y, Tian T, Jin G, Shu Y, Chen Y, Xu L, Zen K, Zhang C, et al: Serum microRNA signatures identified in a genome-wide serum microRNA expression profiling predict survival of non-small cell lung cancer. J Clin Oncol 28: 1721-1726, 2010.

5. Bartel DP: MicroRNAs: Genomics, biogenesis, mechanism, and function. Cell 116: 281-297, 2004.

6. Winter J, Jung S, Keller S, Gregory RI and Diederichs S: Many roads to maturity: MicroRNA biogenesis pathways and their regulation. Nat Cell Biol 11: 228-234, 2009.

7. Rottiers V, Najafi-Shoushtari SH, Kristo F, Gurumurthy S, Zhong L, Li Y, Cohen DE, Gerszten RE, Bardeesy N, Mostoslavsky R, et al: MicroRNAs in metabolism and metabolic diseases. Cold Spring Harb Symp Quant Biol 76: 225-233, 2011.

8. Aigner A: MicroRNAs (miRNAs) in cancer invasion and metastasis: Therapeutic approaches based on metastasis-related miRNAs. J Mol Med Berl 89: 445-457, 2011.

9. Calin GA and Croce CM: MicroRNA signatures in human cancers. Nat Rev Cancer 6: 857-866, 2006. 
10. Esquela-Kerscher A and Slack FJ: Oncomirs - microRNAs with a role in cancer. Nat Rev Cancer 6: 259-269, 2006.

11. Lu J, Getz G, Miska EA, Alvarez-Saavedra E, Lamb J, Peck D, Sweet-Cordero A, Ebert BL, Mak RH, Ferrando AA, et al: MicroRNA expression profiles classify human cancers. Nature 435: 834-838, 2005.

12. Hock R, Furusawa T, Ueda T and Bustin M: HMG chromosomal proteins in development and disease. Trends Cell Biol 17: 72-79, 2007.

13. Shirakawa H, Landsman D, Postnikov YV and Bustin M: NBP-45, a novel nucleosomal binding protein with a tissue-specific and developmentally regulated expression. J Biol Chem 275: 6368-6374, 2000.

14. King LM and Francomano CA: Characterization of a human gene encoding nucleosomal binding protein NSBP1. Genomics 71 163-173, 2001

15. Jiang N, Zhou LQ and Zhang XY: Downregulation of the nucleosome-binding protein 1 (NSBP1) gene can inhibit the in vitro and in vivo proliferation of prostate cancer cells. Asian $\mathrm{J}$ Androl 12: 709-717, 2010.

16. Qu J, Yan R, Chen J, Xu T, Zhou J, Wang M, Chen C, Yan Y and $\mathrm{Lu}$ Y: HMGN5: A potential oncogene in gliomas. J Neurooncol 104: 729-736, 2011.

17. Wahafu W, He ZS, Zhang XY, Zhang CJ, Yao K, Hao H, Song G, He Q, Li XS and Zhou LQ: The nucleosome binding protein NSBP1 is highly expressed in human bladder cancer and promotes the proliferation and invasion of bladder cancer cells Tumour Biol 32: 931-939, 2011.

18. Weng M, Song F, Chen J, Wu J, Qin J, Jin T and Xu J: The high-mobility group nucleosome-binding domain 5 is highly expressed in breast cancer and promotes the proliferation and invasion of breast cancer cells. Tumour Biol 36: 959-966, 2015.

19. Zhou X, Yuan B, Yuan W, Wang C, Gao R and Wang J: The expression and clinical significance of high mobility group nucleosome binding domain 5 in human osteosarcoma. Tumour Biol 35: 6539-6547, 2014.

20. Chen P, Wang XL, Ma ZS, Xu Z, Jia B, Ren J, Hu YX, Zhang QH, Ma TG, Yan BD, et al: Knockdown of HMGN5 expression by RNA interference induces cell cycle arrest in human lung cancer cells. Asian Pac J Cancer Prev 13: 3223-3228, 2012.

21. Barger JF and Nana-Sinkam SP: MicroRNA as tools and therapeutics in lung cancer. Respir Med 109: 803-812, 2015.
22. Zhang XY, Guo ZQ, Ji SQ, Zhang M, Jiang N, Li XS and Zhou LQ: Small interfering RNA targeting HMGN5 induces apoptosis via modulation of a mitochondrial pathway and $\mathrm{Bcl}-2$ family proteins in prostate cancer cells. Asian J Androl 14: 487-492, 2012.

23. Ji SQ, Yao L, Zhang XY, Li XS and Zhou LQ: Knockdown of the nucleosome binding protein 1 inhibits the growth and invasion of clear cell renal cell carcinoma cells in vitro and in vivo. J Exp Clin Cancer Res 31: 22, 2012

24. Yang C, Gao R, Wang J, Yuan W, Wang C and Zhou X: High-mobility group nucleosome-binding domain 5 increases drug resistance in osteosarcoma through upregulating autophagy. Tumour Biol 35: 6357-6363, 2014.

25. Su B, Shi B, Tang Y, Guo Z, Yu X, He X, Li X, Gao X and Zhou L: HMGN5 knockdown sensitizes prostate cancer cells to ionizing radiation. Prostate 75: 33-44, 2015.

26. Kefas B, Comeau L, Floyd DH, Seleverstov O, Godlewski J, Schmittgen T, Jiang J, diPierro CG, Li Y, Chiocca EA, et al: The neuronal microRNA miR-326 acts in a feedback loop with notch and has therapeutic potential against brain tumors. J Neurosci 29: 15161-15168, 2009.

27. Kefas B, Comeau L, Erdle N, Montgomery E, Amos S and Purow B: Pyruvate kinase M2 is a target of the tumor-suppressive microRNA-326 and regulates the survival of glioma cells. Neuro-oncol 12: 1102-1112, 2010.

28. Wang S, Lu S, Geng S, Ma S, Liang Z and Jiao B: Expression and clinical significance of microRNA-326 in human glioma miR-326 expression in glioma. Med Oncol 30: 373, 2013.

29. Zhang ZL, Bai ZH, Wang XB, Bai L, Miao F and Pei HH miR-186 and 326 predict the prognosis of pancreatic ductal adenocarcinoma and affect the proliferation and migration of cancer cells. PLoS One 10: e0118814, 2015.

30. Zhou J, Xu T, Yan Y, Qin R, Wang H, Zhang X, Huang Y, Wang Y, Lu Y, Fu D, et al: MicroRNA-326 functions as a tumor suppressor in glioma by targeting the Nin one binding protein (NOB1). PLoS One 8: e68469, 2013.

31. Wu L, Hui H, Wang LJ, Wang H, Liu QF and Han SX: MicroRNA-326 functions as a tumor suppressor in colorectal cancer by targeting the nin one binding protein. Oncol Rep 33: 2309-2318, 2015

32. Cai M, Wang Z, Zhang J, Zhou H, Jin L, Bai R and Weng Y: Adam17, a target of miR-326, promotes EMT-induced cells invasion in lung adenocarcinoma. Cell Physiol Biochem 36: $1175-1185,2015$ 